\title{
Analysis of "Use of Blood Glucose Meters Featuring Color Range Indicators Improves Glycemic Control and Patients With Diabetes in Comparison to Blood Glucose Meters Without Color (ACCENTS Study)"
}

\author{
Andjela T. Drincic, MD'
}

\begin{abstract}
Self-monitoring of blood glucose is a part of integral care of patients with diabetes mellitus. Understanding and appropriately responding to glucose levels is a fundamental part of self-management. Grady et al's work, published in the current issue of Journal of Diabetes Science and Technology, investigated whether switching people with diabetes from their usual meter to a meter featuring color range indicator (CRI) could improve glycemic control, by facilitating improved understanding of blood glucose targets. In this small but well-designed study, the authors have shown that meters with CRI features offer a potential advantage and may improve glucose control in patients with diabetes, both with TID and T2D, across the therapy spectrum from oral agents to insulin therapy.
\end{abstract}

\section{Keywords}

color range indicators, glucose ranges, blood glucose monitoring, self-monitoring of blood glucose

Self-monitoring of blood glucose (SMBG) is considered to be an integral and critical part of diabetes management. SMBG results facilitate optimal decision making by both patients and their health care professionals (HCP). Based on SMBG, patients can adjust their nutrition or exercise plan and those results can guide medical therapy. The value of SMBG has been undisputed in the management of patients with type 1 diabetes mellitus (T1D), and a correlation has been shown between greater SMBG frequency and lower hemoglobin A1C (A1C). ${ }^{1}$ In those with type 2 diabetes mellitus (T2D) on insulin therapy, more frequent SMBG is associated with meeting A1C goals. ${ }^{2}$ While the use of SMBG in patients with $\mathrm{T} 2 \mathrm{D}$ who are not receiving insulin therapy has been a subject of some controversy, newer data has demonstrated its value. $^{3}$

Currently there are more than 80 blood glucose meters on the market and more are likely to come, offering a wide array of choices. ${ }^{4}$ Most often, the selection of a meter is guided by patients' insurance coverage. In addition, some meters may be better suited to address the special needs of the patient including visual, hearing, or motor skill impairment. Recent technological advancements offer more sophisticated features including wireless Bluetooth or cellular and mobile app connectivity, computer compatibility, and bolus calculation algorithms, to name a few. Accuracy is the main characteristic of a meter known to have an impact on glucose control. Boettcher et al have shown that lower meter accuracy (defined as deviations of SMBG values from the "true" glucose levels) leads to a clinically meaningful increase of hypoglycemic events and had a negative impact on A1C levels. ${ }^{5}$ Similarly, Campos-Nanez et al showed that meters with a large error increased glucose variability and episodes of severe hypoglycemia. The BG monitoring systems with large systematic bias negatively affected $\mathrm{A} 1 \mathrm{C}$ as well. ${ }^{6}$ Thomas et al showed that errors in the blood glucose monitor (BGM) when used for calibration of continuous glucose monitoring (CGM) devices also have a negative impact on detection of hypoglycemia. ${ }^{7}$ Aside from accuracy, little is known if other particular glucometer feature(s) may have an impact on

\footnotetext{
'Department of Internal Medicine: Diabetes, Endocrinology and Metabolism, University of Nebraska Medical Center, Omaha, NE, USA

Corresponding Author:

Andjela T. Drincic, MD, Department of Internal Medicine: Diabetes, Endocrinology and Metabolism, University of Nebraska Medical Center, 984I 20 Nebraska Medical Center, Omaha, NE 68I98-4I20, USA. Email: andjela.drincic@unmc.edu
} 
glucose control. In that respect, Grady et al's study offers much-needed insight, showing that the use of blood glucose meters featuring color range indicator (CRI) can improve glycemic control in patients with diabetes. ${ }^{8}$

This important work is based on recognition of the need to overcome key barriers to appropriate use of SMBG, which can be broadly classified as (1) knowledge (being able to understand SMBG goals and how to achieve them), (2) human factors (knowing how to operate SMBG device), (3) logbook (being able to identify BG patterns by using a logbook), and (4) economic (being able to afford a SMBG device and strips which is closely tied to adequate insurance coverage). ${ }^{3}$ To use SMBG optimally, patients should be able to review, understand, interpret, and respond to their own glucose data. The ability to do so requires understanding of therapy targets, definitions of hypo- and hyperglycemia, and possessing adequate numeracy skills. ${ }^{9}$ While teaching those skills is a key aspect of diabetes education and training, there is limited evidence confirming that indeed patients are adopting the knowledge. On the contrary, a study of 207 patients with $\mathrm{T} 2 \mathrm{D}$, revealed that they were quite tolerant of high BG values as less than a third considered glucose well above 200 $\mathrm{mg} / \mathrm{dl}$ to be high. ${ }^{10}$ In addition, a survey of 866 patients showed that half of them took no action for either low or high BG. ${ }^{11}$ Recognition of these knowledge gaps has led the authors to investigate ways that can help people with diabetes improve their ability to categorize BG in adequate ranges. In their earlier work, the authors have shown that using a CRI can improve the ability of subjects to classify BG as low, in range or high, using three different meters. ${ }^{12}$

In their current work, the authors took this concept one step further and investigated whether switching people with T1D and T2D to a blood glucose meter featuring CRI (OneTouch Verio or OneTouch Verio Flex) could improve glycemic control compared to those remaining on their current meter without color. In this parallel, three-arm, openlabel randomized controlled study involving 163 adults, after 12 weeks, there was a $0.36 \%$ mean $\mathrm{A} 1 \mathrm{C}$ improvement in subjects using meters with CRI compared to the controls. Much of this was driven by those with T1D, showing a $0.5 \%$ mean $\mathrm{A} 1 \mathrm{C}$ improvement compared to the control group.

This unique study confirms not only the value of SMBG but also that the choice of a meter can influence the outcome. Most of the data so far proved the value of SMBG indirectly through increased frequency of testing. In this study, the SMBG frequency did not vary among the three groups. There are multiple other strengths of this study, including multisite and randomized design. No changes in frequency of visits/ contacts with health care providers and medical therapy were made during the course of the study, minimizing potential confounding effects. In addition, the three comparison groups had the same number of patients with T1D and T2D with similar baseline mean $\mathrm{A} 1 \mathrm{C}$ and duration of diabetes.

The weaknesses in this study lie in the small number of subjects within each group making it difficult to understand the difference in the effect size of the two meters with CRI The CRI meters used in this study were OneTouch Verio and OneTouch Verio Flex. They both have the same type of CRI indicating that $\mathrm{BG}$ result is low $(<70 \mathrm{mg} / \mathrm{dl}$ : displayed in blue), in range (70-180 mg/dl: displayed in green), or high ( $>180 \mathrm{mg}$ dl: displayed red). These goals are preset, but can be personalized. The difference between two meters with CRI is that OneTouch Verio also identifies BG patterns and displays related motivational messages. The results of the study suggest that these differences may be meaningful. Namely, contribution of the OneTouch Verio meter use to the reduction of $\mathrm{A} 1 \mathrm{C}$ was greater than that of the OneTouch Verio Flex meter. This was noted both overall, with $0.42 \% \mathrm{~A} 1 \mathrm{C}$ reduction with OneTouch Verio use compared to control, and was particularly pronounced in subjects with T1D where this meter use reduced A1C $0.59 \%$ compared to the control. A1C reductions noted in OneTouch Verio Flex meter users were considered clinically, but not statistically, meaningful. The study was not designed to explore exact mechanisms contributing to the $\mathrm{A} 1 \mathrm{C}$ reduction. Rather, it evaluated the attitudes of subjects and showed that both OneTouch Verio and OneTouch Verio Flex users reported taking more action and having better understanding of diabetes management after switching from their previous meter. In addition, perhaps as expected, the OneTouch Verio users thought it was easier to spot glucose patterns. The novelty lies in the fact that A1C reductions were observed with the use of a CRI meter without increasing the number of daily glucose checks. We do not know if the total insulin dose, carb content, or other factors influencing blood glucose control changed, or if subjects actually bolused insulin more frequently. Interestingly, there were no significant changes in the average glucose or overall glucose ranges despite improvement in $\mathrm{A} 1 \mathrm{C}$ noted in those with CRI meters. This finding can be related to overall low testing frequency, especially in subjects with T2D who on the average tested fewer than twice daily in all groups. More frequent BGM testing including the use of CGM could have shed more light on this issue. In addition, it would be interesting to know if the CRI meter effect observed in this study would be long-lasting, as people in general have a tendency to develop alert and alarm fatigue with time.

In summary, Grady et al have delivered much-needed insight into a value of using meters with CRI. In this small but well-designed study, they have shown that meters with CRI features offer a potential advantage and may improve glucose control in patients with diabetes, both with T1D and $\mathrm{T} 2 \mathrm{D}$, across the therapy spectrum from oral agents to insulin therapy.

\section{Abbreviations}

A1C, hemoglobin A1C; BG, blood glucose; BGM, blood glucose monitor; CGM, continuous glucose monitoring; CRI, color range indicator; HCP, health care professional; SMBG, self-monitoring of blood glucose; T1D, type 1 diabetes mellitus; T2D, type 2 diabetes mellitus. 


\section{Declaration of Conflicting Interests}

The author(s) declared no potential conflicts of interest with respect to the research, authorship, and/or publication of this article.

\section{Funding}

The author(s) received no financial support for the research, authorship, and/or publication of this article.

\section{References}

1. Miller KM, Beck RW, Berenstal RM, et al. Evidence of a strong association between frequency of self-monitoring of blood glucose and hemoglobin A1C levels in T1D exchange clinic registry participants. Diabetes Care. 2013;36(7):20092014.

2. Elgart JF, Gonzalez L, Prestes M, et al. Frequency of self-monitoring blood glucose and attainment of $\mathrm{HbA1C}$ target values. Acta Diabetol. 2016;53(1):57-62.

3. Klonoff DC, Blonde L, Cembrowski G, et al. Consensus report: the current role of self-monitoring of blood glucose in non-insulin-treated type 2 diabetes. J Diabetes Sci Technol. 2011;5(6):1529-1548.

4. Blood glucose meters. Diabetes Forecast. 2018;71(2):61-68.

5. Boettcher C, Dost A, Wudy S, et al. Accuracy of blood glucose meters for self-monitoring affects glucose control and hypoglycemia rate in children and adolescents with type 1 diabetes. Diabetes Technol Ther. 2015;17(4):275-282.
6. Campos-Nanez E, Fortwaengler K, Breton M. Clinical impact of blood glucose monitoring accuracy: an in-silico study. $J$ Diabetes Sci Technol. 2017;11(6):1187-1195.

7. Thomas F, Signal M, Harris D, et al. Continuous glucose monitoring in newborn infants: how do errors in calibration measurements affect detected hypoglycemia? J Diabetes Sci Technol. 2014;8(3):543- 550.

8. Grady M, Katz LB, Levy B. Use of blood glucose meters featuring color range indicators improves glycemic control and patients with diabetes in comparison to blood glucose meters without color (ACCENTS Study) [published online ahead of print May 31, 2018]. J Diabetes Sci Technol. doi:10.1177/1932296818775755.

9. American Diabetes Association. 6. Glycemic targets: standards of medical care in diabetes-2018. Diabetes Care. 2018;41(suppl 1):s55-s64.

10. Evans JM, Mackison D, Swanson V, et al. Self-monitoring of blood glucose in type 2 diabetes: patients' perceptions of "high" readings. Diabetes Res Clin Pract. 2013;102(1):e5-e7.

11. Polonsky WH, Fisher L, Hessler D, et al. A survey of blood glucose monitoring in patients with type 2 diabetes: are recommendations from health care professionals being followed? Curr Med Res Opin. 2011;27(suppl 3):31-37.

12. Grady M, Katz LB, Cameron H, et al. A comprehensive evaluation of a novel color range indicator in multiple blood glucose meters demonstrates improved glucose range interpretation and awareness in subjects with type 1 and type 2 diabetes. $J$ Diabetes Sci Technol. 2016;10(6):1324-1332. 